\title{
Thermal X-Ray Line Emission from Accreting Black Holes
}

\section{Citation}

Narayan, Ramesh, and John Raymond. 1999. "Thermal X-Ray Line Emission from Accreting Black Holes." The Astrophysical Journal 515 (2): L69-72. https://doi.org/10.1086/311973.

\section{Permanent link}

http://nrs.harvard.edu/urn-3:HUL.InstRepos:41384921

\section{Terms of Use}

This article was downloaded from Harvard University's DASH repository, and is made available under the terms and conditions applicable to Other Posted Material, as set forth at http:// nrs.harvard.edu/urn-3:HUL.InstRepos:dash.current.terms-of-use\#LAA

\section{Share Your Story}

The Harvard community has made this article openly available.

Please share how this access benefits you. Submit a story.

\section{Accessibility}




\title{
Thermal X-Ray Line Emission from Accreting Black Holes
}

\author{
Ramesh Narayan and John Raymond \\ Harvard-Smithsonian Center for Astrophysics, 60 Garden St., Cambridge, MA 02138
}

\begin{abstract}
We present model X-ray spectra of accreting black holes with advection-dominated accretion flows, paying attention to thermal emission lines from the hot plasma. We show that the Advanced X-ray Astrophysical Facility (AXAF) might be able to observe lines from X-ray binaries such as V404 Cyg in quiescence, the Galactic center black hole Sagittarius $A^{*}$, and the nuclei of nearby galaxies such as M87. Line intensities can provide new diagnostics to study the accreting plasma in these and related systems.

Subject headings: Accretion, Black Holes, Galaxies: Nuclei, Galaxy: Center, Radiation Mechanisms: Thermal, X-Rays: Binaries, X-Rays: Spectra
\end{abstract}

\section{Introduction}

The AXAF satellite will enable us to measure X-ray spectra of astronomical sources with good spectral resolution - about $60 \mathrm{eV}$ at $1 \mathrm{keV}$ for the AXAF CCD Imaging Spectrometer, ACIS - and excellent spatial resolution (better than 1 arcsecond). With an effective area of $\sim 300 \mathrm{~cm}^{2}$, ACIS should observe spectral lines from a number of X-ray sources. The XMM satellite may provide even greater sensitivity for lines. Measurements of line intensities will provide important new diagnostics for the physical conditions of the radiating plasma.

Some of the most important X-ray sources in the sky are accreting black holes. Examples are stellar mass black holes in X-ray binaries, and supermassive black holes in the nucleus of our Galaxy and other galaxies. X-ray emission from these sources arises as a result of optically thick thermal radiation from an accretion disk (cf. Tanaka \& Shibazaki 1996), or by Compton-scattering of soft photons by a hot optically thin plasma, either in a corona (e.g. Haardt \& Maraschi 1991) or in an advection-dominated accretion flow (ADAF, Narayan \& Yi 1994, 1995, Abramowicz et al. 1995, see Narayan, Mahadevan \& Quataert 1998b and Kato, Fukue \& Mineshige 1998 for reviews), or by thermal bremsstrahlung from hot low density gas. The spectral energy distributions corresponding to these different processes have been studied by a number of authors (see Liang 1998 and Mushotzky, Done \& Pounds 1993 for reviews).

Fewer models are available for X-ray line emission. Fluorescent line emission from cool gas irradiated by a hot corona has been studied in some detail (Mushotzky et al. 1993, Tanaka et al. 1995), but thermal $\mathrm{X}$-ray line emission from hot optically thin gas around black holes has not been discussed very much. In the present Letter, we show that black holes that accrete via ADAFs with relatively low mass accretion rates might produce X-ray lines detectable with AXAF or XMM. These lines could provide new constraints on the accretion flows in these systems. We point out three significant diagnostic possibilities. First, the equivalent widths of the emission lines increase with the size of the ADAF region. Second, for a given ADAF size the

\footnotetext{
${ }^{1}$ rnarayan@cfa.harvard.edu

2 jraymond@cfa.harvard.edu
} 
equivalent widths are much larger for models that include a wind than for models with no wind. And, third, photoionization is unimportant in an ADAF, while it dominates in some accretion disk corona models.

\section{ADAF Models}

Both corona models and ADAF models feature hot optically thin gas, and both lead to thermal X-ray emission. However, corona models are usually very Compton-dominated, so that any thermal lines are likely to have low equivalent widths. ADAFs too are Compton-dominated when the mass accretion rate is high; if the Eddington-scaled accretion rate, $\dot{m} \equiv \dot{M} / 2.2 \times 10^{-8} m M_{\odot} \mathrm{yr}^{-1}$ (where $m$ is the black hole mass in solar units), is close to a critical value $\dot{m}_{\text {crit }} \sim 0.05-0.1$ (Esin, McClintock \& Narayan 1997), the Comptonization of soft photons (either from an outer disk or from thermal synchrotron emission) is so strong that it greatly exceeds the thermal emission.

As the mass accretion rate in an ADAF decreases to $\dot{m} \lesssim 0.01$, thermal bremsstrahlung becomes relatively more important (Esin et al. 1997); thermal lines should then have measureable equivalent widths. The electron temperature in an ADAF varies roughly as $T_{e} \sim 10^{12} \mathrm{~K} / r$ for $r \gtrsim 10^{3}$ (Narayan \& Yi 1995), where $r$ is the radius in Schwarzschild units $\left(2.95 \times 10^{5} \mathrm{~m} \mathrm{~cm}\right)$. Since the strongest X-ray line emission arises from thermal gas with $T_{e} \sim 10^{7}-10^{8} \mathrm{~K}$, the region of the flow between $r \sim 10^{4}-10^{5}$ is most important for our purpose. ADAFs can extend to such large radii only for low $\dot{m}$ (Narayan \& Yi 1995, Narayan et al. 1998b), another reason to concentrate on low- $\dot{m}$ systems.

We have computed ADAF models and corresponding X-ray spectra of the following three low- $\dot{m}$ systems: the X-ray binary V404 Cyg in quiescence, the Galactic Center source Sgr A*, and the nucleus of the galaxy M87. The methods are described in Quataert \& Narayan (1998, and references therein). ADAF models are not unique, as there is considerable uncertainty associated with possible winds (Narayan \& Yi 1994, Blandford \& Begelman 1998, Quataert \& Narayan 1998). Therefore, for each of our three systems we have computed two models, one in which there is no wind (referred to as NW, the accretion rate $\dot{m}$ is taken to be independent of radius), and one with a moderately strong wind (referred to as $\mathrm{W}, \dot{m}$ is assumed to vary with radius as $\left.r^{0.4}\right)$. These two models probably bracket the true situation. We assume that in the NW model the electrons in the accreting plasma receive only a fraction $\delta=0.01$ of the viscously dissipated heat in the accretion flow. However, in the W model we set $\delta=0.3$, as recommended by Quataert \& Narayan (1998), in order to fit the continuum spectrum. We assign reasonable values to the other microscopic parameters: viscosity parameter $\alpha=0.1$, plasma $\beta=10$. The results are not very sensitive to the values of these parameters.

The system-specific parameters are as follows: see Narayan, Barret \& McClintock (1997) for a compilation of the data on V404 Cyg, Narayan et al. (1998a) for Sgr A*, and Reynolds et al. (1996) for M87. The black hole masses are $m=12(\mathrm{~V} 404 \mathrm{Cyg}), m=2.5 \times 10^{6}\left(\mathrm{Sgr} \mathrm{A}^{*}\right), m=3 \times 10^{9}(\mathrm{M} 87)$, the distances are $3.5 \mathrm{kpc}(\mathrm{V} 404 \mathrm{Cyg}), 8.5 \mathrm{kpc}\left(\mathrm{Sgr} \mathrm{A} \mathrm{A}^{*}\right), 16 \mathrm{Mpc}(\mathrm{M} 87)$, and the absorbing columns are $N_{H}=1.1 \times 10^{22} \mathrm{~cm}^{-2}$ (V404 Cyg), $N_{H}=6 \times 10^{22} \mathrm{~cm}^{-2}\left(\mathrm{Sgr} \mathrm{A} \mathrm{A}^{*}\right), N_{H}=2.5 \times 10^{20} \mathrm{~cm}^{-2}$ (M87, this $N_{H}$ includes only the contribution of our Galaxy). The mass accretion rates are not known a priori. We fit $\dot{m}$ in each model so as to reproduce the observed X-ray continuum. This gives $\dot{m}=0.0010,0.0060$ (NW and W models of V404 Cyg), $0.000070,0.00024$ (Sgr A*), 0.0010, 0.011 (M87). For the $\mathrm{W}$ models, where $\dot{m}$ varies with $r$, the quoted $\dot{m}$ corresponds to the outer edge of the ADAF.

The greatest uncertainty in the models has to do with the size of the ADAF region. In V404 Cyg, the ADAF is believed to extend from the black hole horizon $(r=1)$ to a transition radius $r_{t r}$, beyond which the 
flow consists of a thin disk plus a corona. We take $r_{t r}=10^{4.4}$, as indicated by the width of the $H_{\alpha}$ line from the outer disk (Narayan et al. 1997). In the coronal region, we set $\dot{m} \propto r_{t r} / r$ to model the evaporation of gas from the disk into the corona (cf. Esin et al. 1997). In Sgr $A^{*}$, there is no evidence for an outer disk, so we consider a pure ADAF model extending from $r=1$ to an outer radius $r_{\text {out }}$. The stars whose winds supply most of the accreting mass (cf. Coker \& Melia 1997) are located at $r \gtrsim$ few $\times 10^{5}$. We therefore set $r_{\text {out }}=10^{5}$. For simplicity, we use the same value of $r_{\text {out }}$ for M87 as well.

\section{Model X-Ray Line Spectra}

Each quasi-spherical ADAF model gives the electron density $n_{e}$ and electron temperature $T_{e}$ as a function of $r$ on a radial grid with ten zones per decade of $r$. Figure 1 shows these quantities for the NW and $\mathrm{W}$ models of Sgr $\mathrm{A}^{*}$.

For calculating the line spectrum, we divide the flow into an inner region from $r=1-10^{2}$ and an outer region beyond $10^{2}$. We compute the continuum emission from the inner region due to synchrotron, bremsstrahlung and Comptonization, but we do not compute line emission; this is reasonable since the temperature is $>10^{9} \mathrm{~K}$ (Fig. 1), and the astrophysically abundant elements are fully ionized. In the outer region, we compute the line emission and thermal continuum in detail, but we do not consider either synchrotron emission or the effect of Comptonization; these latter processes are very steep functions of the temperature, and are negligible outside $r=10^{2}$. The spectra we present include only the radiation from the accreting gas; we ignore any emission by the wind.

The spectral calculation in the outer region employs an updated version of the Raymond \& Smith (1977) $\mathrm{X}$-ray code to compute the bremsstrahlung, recombination and two-photon continua and the emission in spectral lines. For the present models we are mostly concerned with H-like and He-like ions. Collisional excitation (e.g. Pradhan, Norcross \& Hummer 1981; Pradhan 1985), recombination to excited levels (e.g. Mewe, Schrijver \& Sylwester 1980) and dielectronic recombination satellite lines (e.g. Dubau et al 1981; Bely-Daubau et al 1982) are included. The calculation has been done with a spectral binning of $60 \mathrm{eV}$ over a range of 0.2-30 keV. We have ignored Doppler smearing of the lines, which for an ADAF is of order the thermal broadening, and is relatively unimportant at the large radii of interest.

The calculations proceed outward from the innermost zone at $r=10^{2}$. In each zone, we use the local density and temperature to compute the thermal emission. We include photoionization by all radiation emitted at smaller radii (including the continuum emission from the region inside $r=10^{2}$ ). However, we find that photoionization affects the ionization state of the abundant ions by at most a few per cent. This results from the relatively inefficient conversion of accretion energy to X-ray luminosity in an ADAF $(<0.1 \%$ in the models presented here). It stands in contrast to the dominance of photoionization in the coronae of Low Mass X-ray Binaries, which convert $\sim 20 \%$ of the accretion energy to X-rays (Raymond 1993; Ko \& Kallman 1994). We have not included photoabsorption, but we confirmed a posteriori that it is not important in reducing the X-ray flux.

\section{Results}

Figure 2 shows model X-ray spectra of V404 Cyg, Sgr A* and M87, for NW models (upper panel) and W models (lower panel); the spectra have been corrected for absorption using the estimated $N_{H}$. The ordinate 
shows the photon count rate per $60 \mathrm{eV}$ bin in units of $\mathrm{cm}^{-2} \mathrm{~s}^{-1}$. For the ACIS detector, with an effective area of $\sim 300 \mathrm{~cm}^{2}$, and an integration time of $3 \times 10^{4} \mathrm{~s}$, the number of counts we may expect to detect in an observation is the photon count rate multiplied by $\sim 10^{7} \mathrm{~cm}^{2} \mathrm{~s}$. We see from Fig. 2 that several of the lines in the model spectra could be observed with good signal to noise in such an observation.

Table 1 lists equivalent widths of a few selected lines. In general, we expect to see lines of H-like and He-like ions of high- $Z$ elements. Figure 3 shows the equivalent widths of a few lines as a function of outer radius for the Sgr A* model with a wind. The equivalent width of each line rises as the temperature declines to the characteristic temperature at which the line is formed. As the temperature falls still lower, there is little emission in either the line or the continuum at that energy, and the equivalent width remains constant. Most lines form at fairly large radii, $r \sim 10^{4}-10^{5}$. Clearly, by studying the relative intensities of different lines, it would be possible to estimate how much gas is present at different temperatures; moreover, because of the near one-to-one mapping between temperature and radius at the radii of interest (see Fig. 1), we could estimate the run of gas density with radius.

Both Fig. 2 and Table 1 show a striking difference between non-wind (NW) and wind (W) models, with the latter producing significantly stronger lines. NW models are typically more Compton-dominated than W models (Quataert \& Narayan 1998), and therefore have less thermal emission. Also, because $\dot{m}$ is a rising function of $r\left(\dot{m} \propto r^{0.4}\right)$, W models have relatively more gas at larger radii (see Fig. 1). Since the bulk of the line emission comes from radii outside about $10^{4}$ (Fig. 3), this dramatically enhances the line emission in these models.

\section{Discussion}

The main result of this Letter is that high resolution X-ray spectra of low-luminosity accreting black

holes could reveal interesting emission lines if the accretion in these sources occurs via an ADAF. Some of the X-ray lines could be observed with good signal to noise with the ACIS detector on AXAF, and with XMM, ASTRO E or the proposed Constellation-X.

Detection of X-ray lines with any reasonable strength would imply an ADAF with a large outer radius, and would rule out a Compton-dominated corona model. Also, models with winds have significantly larger equivalent widths than those without. As Fig. 3 shows, different lines arise from different radii. Therefore, if the strengths of many lines are measured, both the size of the ADAF and the presence or absence of a wind could be determined. In principle, the composition of the gas could also be estimated.

In addition, the models predict that photoionization is unimportant, which is a unique feature of the ADAF model and distinguishes it from corona models. There are several X-ray diagnostics with which it should be possible to distinguish collisionally ionized hot plasma from photoionized gas (Liedahl et al 1992; Kallman 1995). For the present case, the most promising diagnostic would be the ratio of the $1 \mathrm{~s}^{2}-1 \mathrm{~s} 2 \mathrm{p}^{1} \mathrm{P}$ resonance line to the $1 \mathrm{~s}^{2}-1 \mathrm{~s} 2 \mathrm{~s}^{3} \mathrm{~S}$ forbidden line in any of the He-like ions (e.g. the $\lambda 1.850 \AA$ and $\lambda 1.867 \AA$ lines of Fe XXV).

We should emphasize that the models presented in this Letter are only illustrations of typical effects that one may expect in sources with ADAFs. In the wind models, for instance, we calculate only the line emission from the accreting gas and ignore any radiation from the wind. In addition, there could be line emission from diffuse hot gas surrounding the source (e.g. in Sgr A*, Koyama et al. 1996), though such emission is not a serious problem with an instrument like ACIS which has excellent angular resolution. 
Of the models discussed here, those of V404 Cyg are perhaps the most secure. It is the only one of the three sources for which the X-ray luminosity is known accurately (Narayan et al. 1997), and for which we have a reliable estimate of $\dot{m}$. The size of the ADAF zone is also reasonably constrained. However, the model uses a simplified description of the coronal gas above the outer thin disk.

The situation is worse for the other two sources. The X-ray emission from the accretion flow in Sgr A* is not well constrained, both because of the poor angular resolution of current observations which cannot distinguish the black hole from the surrounding diffuse gas (Koyama et al. 1996), and because of the large uncertainty in the absorbing column to the source. Both problems can be solved with ACIS observations. If the X-ray continuum is not much below the estimate used by Narayan et al. (1998a), then Fig. 2 indicates that line emission would be relatively easy to observe.

In the case of M87, it is not clear that the observed X-rays (Reynolds et al. 1996) necessarily come from the accretion flow onto the central black hole. The size of the ADAF too is highly uncertain; $r_{\text {out }}$ could have any value from $10^{2}-10^{5}$. Observations with AXAF will clarify the situation. In an optimistic scenario (lower panel of Fig. 2), lines should be seen readily.

Acknowledgments. We thank Josh Grindlay for useful discussions on the capabilities of AXAF and XMM, and Jeff McClintock, Kristen Menou and Eliot Quataert for comments on the manuscript. This work was supported in part by NASA grants NAG 5-2837 and 5-2845 to the Smithsonian Astrophysical Observatory.

\section{References}

Abramowicz, M., Chen, X., Kato, S., Lasota, J.-P., \& Regev, O., 1995. ApJ. 438. L37

Blandford, R. D., \& Begelman, M. C., 1998, MNRAS submitted astro-ph/9809083)

Bely-Daubau, F., Faucher, P., Dubau, J., \& Gabriel, A.H. 1982, MNRAS, 198, 239

Coker, R. F., \& Melia, F. 1997, ApJ, 488, L149

Dubau, J., Loulergue, M., Gabriel, A.H., Steenman-Clark, L., \& Volonte, S. 1981, MNRAS, 195, 705

Esin, A. A., McClintock, J. E., \& Narayan, R. 1997, ApJ, 489, 867

Haardt, F., \& Maraschi, L. 1991, ApJ, 380, L51

Kallman, T.R. 1995, ApJ, 455, 603

Kato, S., Fukue, J., \& Mineshige, S. 1998, Black-Hole Accretion Disks (Kyoto: Kyoto Univ. Press)

Ko, Y.-K., \& Kallman, T.R. 1994, ApJ, 431, 273

Koyama, K. et al. 1996, PASJ, 48, 249

Liang, E. P. 1998, Phys. Repts., 302, 67

Liedahl D.A., Kahn, S.M., Osterheld, A.L., \& Goldstein, W.H. 1992, ApJ, 391, 306

Mewe, R., \& Schrijver, J., \& Sylwester, J. 1980, A\& AS, 40, 323

Mushotzky, R. F., Done, C., \& Pounds, K. A. 1993, ARA\&A, 31, 717

Narayan, R., Barret, D., \& McClintock, J. E. 1997, ApJ, 482, 448

Narayan, R., Mahadevan, R., Grindlay, J. E., Popham, R. G., \& Gammie, C. 1998a, ApJ, 492, 554

Narayan, R., Mahadevan, R., \& Quataert, E. 1998b, The Theory of Black Hole Accretion disks, eds. M. A. Abramowicz, G. Bjornsson, \& J. E. Pringle (Cambridge Univ. Press), in press astro-ph/9803141

Narayan, R., \& Yi, I. 1994, ApJ, 428, L13

Narayan, R., \& Yi, I. 1995, ApJ, 452, 710

Pradhan, A.K. 1985, ApJ Suppl., 59, 183

Pradhan, A.K., Norcross, D.W., \& Hummer, D.G. 1981, ApJ. 246. 1031

Quataert, E., \& Narayan, R. 1998, ApJ, sumitted (astro-ph/9810136)

Raymond, J.C., \& Smith, B.W. 1977, ApJ Suppl., 35, 419

Raymond, J.C. 1993, ApJ, 412, 267

Reynolds, C. S., Di Matteo, T., Fabian, A. C., Hwang, U., \& Canizares, C. R. 1996, MNRAS, 283, L111

Tanaka, Y., \& Shibazaki, N. 1996, ARA\&A, 34, 607

Tanaka, Y. et al. 1995, Nature, 375, 659 
Table 1. Equivalent widths (in eV) of some X-ray lines in ADAF models of V404 Cyg with No Wind (VNW) and with Wind (VW), Sgr A* (SNW, SW), and M87 (MNW, MW).

\begin{tabular}{lccccccc}
\hline \hline \multicolumn{1}{c}{ Ion } & $\lambda(\AA)$ & VNW & VW & SNW & SW & MNW & MW \\
\hline O VII & 21.60 & 0.0 & 0.0 & 0.0 & 0.6 & 0.0 & 0.6 \\
O VIII & 18.97 & 0.0 & 1.3 & 3.1 & 47.5 & 0.2 & 47.2 \\
Mg XI & 9.168 & 0.0 & 0.1 & 0.3 & 5.6 & 0.0 & 5.4 \\
Mg XII & 8.425 & 0.0 & 1.4 & 2.6 & 31.0 & 0.2 & 30.6 \\
Si XIII & 6.648 & 0.0 & 0.8 & 2.4 & 37.4 & 0.2 & 36.3 \\
Si XIV & 6.180 & 0.1 & 6.0 & 8.2 & 79.8 & 0.6 & 79.1 \\
S XV & 5.039 & 0.0 & 1.8 & 3.2 & 40.4 & 0.2 & 39.4 \\
S XVI & 4.727 & 0.1 & 7.2 & 6.7 & 50.1 & 0.5 & 49.8 \\
Ar XVII & 3.949 & 0.0 & 2.9 & 3.0 & 31.0 & 0.2 & 30.3 \\
Ar XVIII & 3.731 & 0.1 & 7.1 & 4.8 & 28.4 & 0.4 & 28.3 \\
Ca XIX & 3.173 & 0.0 & 1.7 & 1.1 & 10.1 & 0.1 & 9.8 \\
Ca XX & 3.020 & 0.0 & 2.7 & 1.6 & 7.8 & 0.1 & 7.8 \\
Fe XXIV & 1.861 & 0.0 & 1.6 & 0.7 & 7.5 & 0.1 & 7.3 \\
Fe XXV & 1.850 & 1.0 & 110 & 50.9 & 327 & 3.6 & 315 \\
Fe XXV & 1.867 & 0.5 & 41.7 & 19.5 & 124 & 1.6 & 121 \\
Fe XXV & 1.859 & 0.5 & 43.5 & 19.3 & 134 & 1.5 & 131 \\
Fe XXV & 1.590 & 0.2 & 23.0 & 10.2 & 70.0 & 0.7 & 67.1 \\
Fe XXV & 1.855 & 0.2 & 35.0 & 15.5 & 160 & 1.2 & 155 \\
Fe XXVI & 1.780 & 1.7 & 79.1 & 60.5 & 190 & 4.7 & 189 \\
Fe XXVI & 1.500 & 0.4 & 16.4 & 13.7 & 40.4 & 1.1 & 40.6 \\
Ni XXVII & 1.587 & 0.1 & 6.4 & 3.1 & 18.0 & 0.2 & 17.3 \\
Ni XXVIII & 1.530 & 0.1 & 3.9 & 3.4 & 9.4 & 0.3 & 9.5 \\
\hline & & & & & & & \\
\hline
\end{tabular}



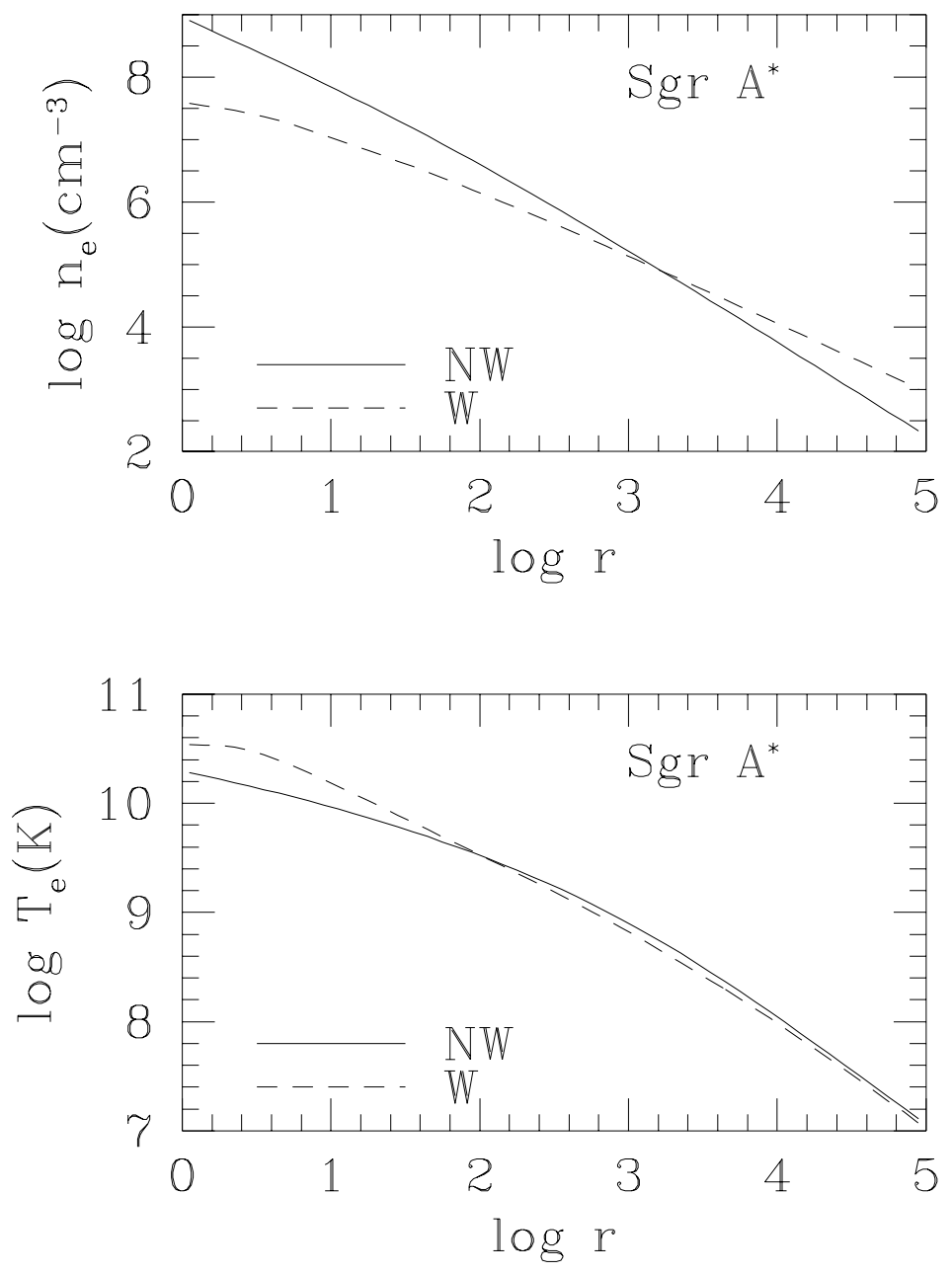

Fig. 1.- Electron density $n_{e}$ and electron temperature $T_{e}$ versus radius $r$ (in Schwarzschild units) for two models of Sgr $\mathrm{A}^{*}$. NW refers to a model with no wind ( $\dot{m}$ independent of $r$ ), and $\mathrm{W}$ to one with a wind $\left(\dot{m} \propto r^{0.4}\right)$. 

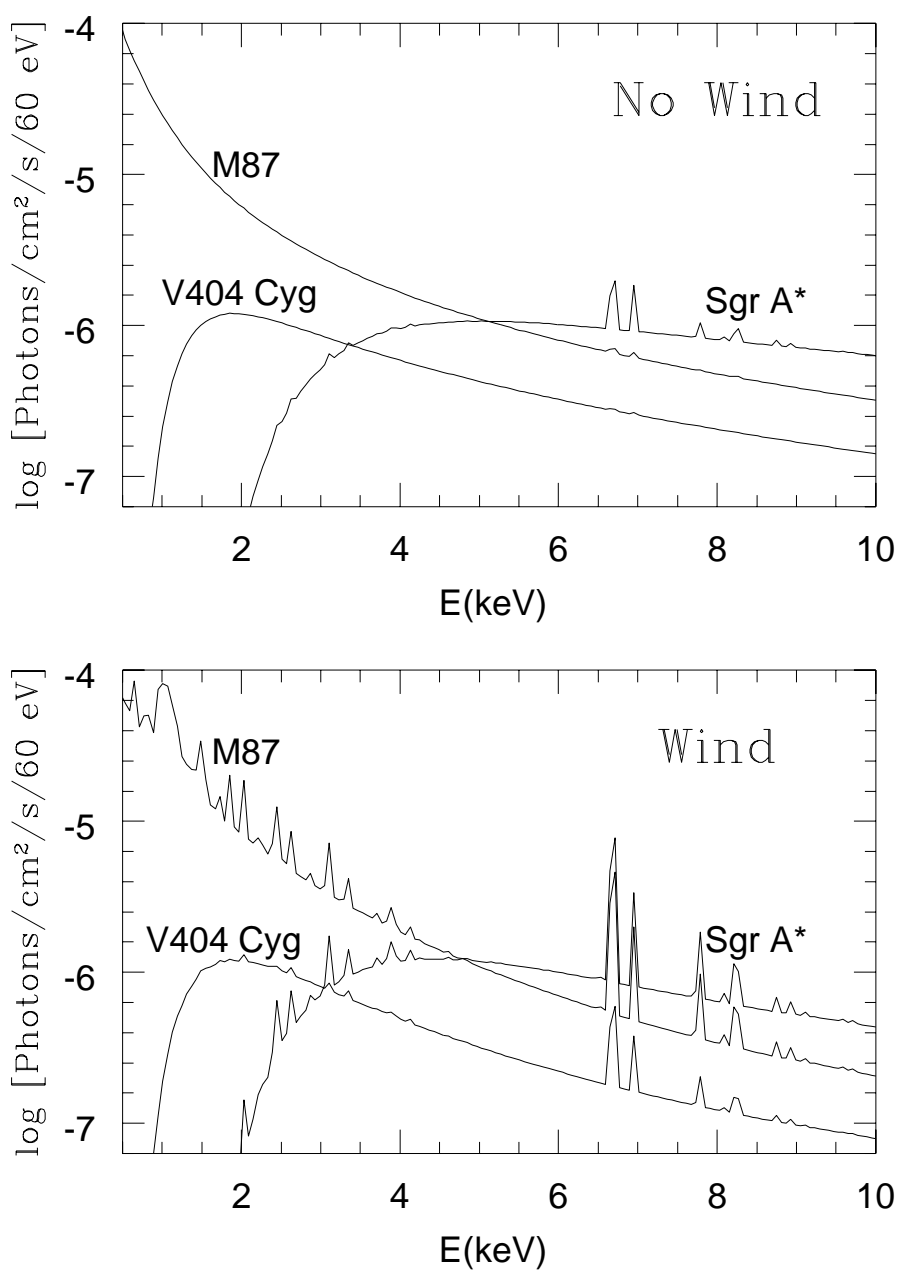

Fig. 2.- Upper panel: Model X-ray line spectra of V404 Cyg (in quiescence), Sgr A*, and the nucleus of M87 (the spectrum of M87 is shifted downward by 0.3 in the log for clarity). The models assume that the accretion occurs via an ADAF with no mass loss to a wind. The spectra are shown with energy bins of $60 \mathrm{eV}$ and have been corrected for interstellar absorption with the appropriate values of $N_{H}$. Lower panel: Corresponding models for the case when there is a moderately strong wind: $\dot{m} \propto r^{0.4}$. 


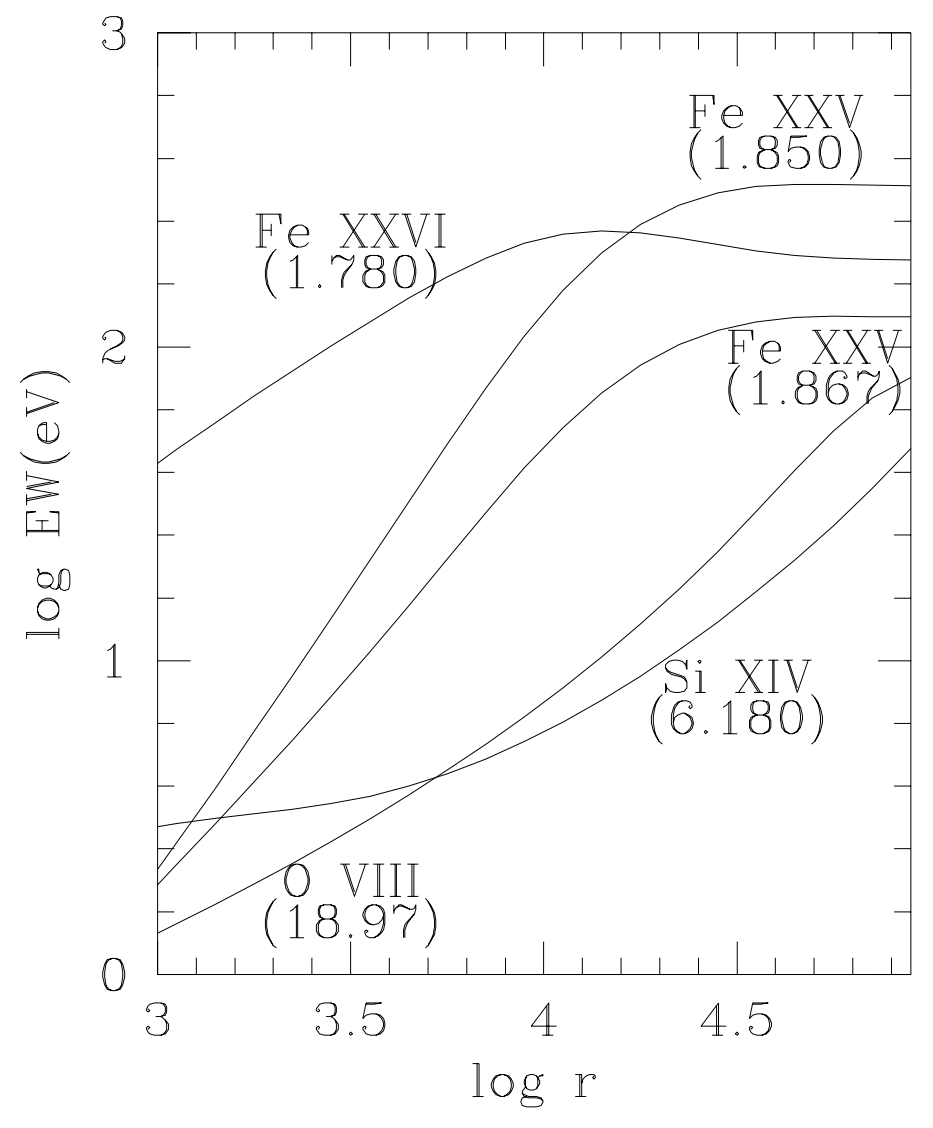

Fig. 3.- Variation with radius $r$ of the equivalent widths of selected X-ray lines (identified by wavelength in $\AA$ ) in the model of Sgr $\mathrm{A}^{*}$ with a wind. Note that different lines saturate at different radii, so that by studying relative line intensities it should be possible to determine the amount of gas present at different radii. 